OPEN ACCESS

Edited by:

Jing Zhao,

University of Connecticut,

United States

Reviewed by:

Yugang Sun,

Temple University, United States

Xiaoji Xie,

Nanjing Tech University, China

*Correspondence:

Kun Liu

kliu@jlu.edu.cn

Specialty section:

This article was submitted to

Nanoscience,

a section of the journal

Frontiers in Chemistry

Received: 08 June 2018

Accepted: 19 July 2018

Published: 10 August 2018

Citation:

Chang $Y-X$, Gao H-M, Zhang N-N,

Tao X-F, Sun T, Zhang J, Lu Z-Y, Liu K

and Yang B (2018) Synergistic

Reducing Effect for Synthesis of Well-Defined Au Nanooctopods With

Ultra-Narrow Plasmon Band Width

and High Photothermal Conversion

Efficiency. Front. Chem. 6:335.

doi: 10.3389/fchem.2018.00335

\section{Synergistic Reducing Effect for Synthesis of Well-Defined Au Nanooctopods With Ultra-Narrow Plasmon Band Width and High Photothermal Conversion Efficiency}

\author{
Yi-Xin Chang ${ }^{1}$, Hui-Min Gao ${ }^{2}$, Ning-Ning Zhang ${ }^{1}$, Xing-Fu Tao ${ }^{1}$, Tianmeng Sun ${ }^{3}$, \\ Junhu Zhang ${ }^{1,4}$, Zhong-Yuan Lu ${ }^{1,2}$, Kun Liu ${ }^{1,4 *}$ and Bai Yang ${ }^{1,4}$ \\ ' State Key Laboratory of Supramolecular Structure and Materials, College of Chemistry, Jilin University, Changchun, China, \\ ${ }^{2}$ Laboratory of Theoretical and Computational Chemistry, Institute of Theoretical Chemistry, Jilin University, Changchun, \\ China, ${ }^{3}$ The First Bethune Hospital and Institute of Immunology, Jilin University, Changchun, China, ${ }^{4}$ State Key Laboratory of \\ Applied Optics, Changchun Institute of Optics, Fine Mechanics and Physics, Chinese Academy of Sciences, Changchun, \\ China
}

Branched Au nanoparticles have attracted intense interest owing to their remarkable properties and a wide variety of potential applications in surface-enhanced Raman spectroscopy (SERS), photothermal therapy, photoacoustic imaging, and biomedicines. The morphology and spatial arrangement of branches play the most crucial role in the determination of their properties and applications. However, it is still a synthetic challenge to control the exact arm numbers of branches with specific spatial arrangements. Here we report a facile method for the kinetically controlled growth of Au nanooctopods (NOPs) with a high yield (81\%), monodispersity, and reproducibility by using the synergistic reducing effect of ascorbic acid and 1-methylpyrrolidine. The NOPs have eight arms elongated along $<111>$ directions with uniform arm lengths. Due to their well-defined size and shape, NOPs show ultra-narrow surface plasmon band width with a full width at half maximum of only $76 \mathrm{~nm}(0.20 \mathrm{eV})$. Upon irradiation with laser, the NOPs possessed excellent photothermal conversion efficiencies up to $83.0 \%$ and photoacoustic imaging properties. This work highlights the future prospects of using NOPs with desired physicochemical properties for biomedical applications.

Keywords: nanooctopods, nanobranch, nanostar, ultra-narrow plasmon band width, photothermal conversion efficiency, photoacoustic imaging, synergistic reducing effect

\section{INTRODUCTION}

Research on Au nanoparticles has been boosted by diverse applications because of their unique size- and shape-dependent properties (Link and El-Sayed, 2000; Kelly et al., 2003; Li et al., 2014; Boles et al., 2016). The past decade has witnessed spectacular success in developing a myriad of methods for shape-controlled synthesis of anisotropic Au nanoparticles with well-defined structures, such as triangles (Malikova et al., 2002), prisms (Shankar et al., 2004), rods (Jana et al., 2001; Nikoobakht and El-Sayed, 2003), cubes (Sun and Xia, 2002; Kim et al., 2004; Zhang, J. et al., 2010), shells (Averitt et al., 1997), stars (Hao et al., 2004; Nehl et al., 2006; Kumar et al., 2007), 
bipyramids (Liu and Guyot-Sionnest, 2005), cages (Skrabalak et al., 2008), etc. Among them, Au nanobranches with a center core and several protruding arms with sharp tips have drawn enormous attention due to their excellent localized surface plasmon resonance (LSPR) properties (Chen et al., 2003; Guerrero-Martínez et al., 2011; Lim and Xia, 2011; Ye et al., 2015). The arm-length dependent LSPR band in the biological window (650-1,350 nm), remarkable enhanced local E-field at their sharp tips, and relative high cellular uptake and low cytotoxicity make Au nanobranches very attractive for bioapplications, such as SERS-based sensing (Khoury and Vo-Dinh, 2008; Indrasekara et al., 2014), photoacoustic imaging (Wei et al., 2009; Cheng et al., 2014), photothermal therapy (Yuan et al., 2012; Wang et al., 2013), and nanomedicines (Dam et al., 2012), etc.

Many of these applications rely on single wavelength laser to excite the plasmon resonance. To maximize the radiation efficiency and minimize the toxicity and negative side effect in bio-applications, $\mathrm{Au}$ nanobranches with narrow plasmon band matching with the laser wavelength is strongly desired. In addition, a narrower plasmon peak width implies a smaller plasmon damping and a larger local electric field enhancement of the nanoparticles (Sönnichsen et al., 2002). Narrow plasmon linewidth of nanoparticles is vital in many plasmonic applications (Zhu et al., 2016), such as monitoring their electron transfer to other materials (Hoggard et al., 2013), studying chemical bonding on their surface (Zijlstra et al., 2012), and developing ultrasensitive chemical and biological LSPR sensors (Mayer and Hafner, 2011). Generally, the extinction spectra of nanobranches, however, exhibit very broad UV-Vis-NIR peaks (inhomogeneous broadening effects) due to unavoidable shape differences of individual $\mathrm{Au}$ nanobranches, that is, the number, size, and orientation of their arms vary greatly among them. Although $\mathrm{Au}$ nanooctopods can be synthesized with the cubic Au seeds from previous literature protocols, the arms of the octopods were too short to exhibit highly branched architectures and sharp tips. In addition, the Au nanooctopods showed broad band due to their size and structural inhomogeneity (Smith et al., 2016). Therefore, the lack of reliable synthetic methods for highly branched Au nanoparticles with accurate control of the number of arms, spatial arrangement of arms, arm size, high yield as well as reproducibility hinders the fundamental understanding of their properties and the assessment of their potentials (Niu et al., 2015).

Herein, we report a facile kinetically controlled growth of monodisperse NOPs with a high yield and narrow LSPR band by using 1-methylpyrrolidine (1-MP) and ascorbic acid (AA) as effective co-reducing agents for $\mathrm{HAuCl}_{4}$ at room temperature. With the presence of single-crystalline $\mathrm{Au}$ seeds, the high reduction rate of $\mathrm{Au}$ precursor leads to the growth of eight arms along $<111>$ directions of the seeds. The NOPs show strong and ultra-narrow LSPR peak in the range of 660-720 nm. They exhibit remarkable photothermal conversion efficiencies up to $83.0 \%$ and a photoacoustic response upon $680 \mathrm{~nm}$ laser irradiation. These NOPs hold great promise of highly efficient photothermal conversion and photoacoustic dual-functional agents.

\section{MATERIALS AND METHODS}

\section{Materials}

Hexadecyltrimethylammonium bromide (CTAB), 1methylpyrrolidine (1-MP), gold (III) chloride trihydrate $\left(\mathrm{HAuCl}_{4} \cdot 3 \mathrm{H}_{2} \mathrm{O}\right)$, ascorbic acid (AA), and sodium borohydride $\left(\mathrm{NaBH}_{4}\right)$ were purchased from Sigma-Aldrich. Monomethoxypoly (ethylene glycol)-thiol (mPEG-SH, molecular weight = $5,000 \mathrm{~g} / \mathrm{mol}$ ) was purchased from JenKem Technology. All the reagents were used as received without further purification. Deionized water $(18.2 \mathrm{M} \Omega \cdot \mathrm{cm})$ was used for all solution preparations.

\section{Synthesis of Au Seeds}

The gold seed nanoparticles were prepared according to the method reported previously with a slight modification (Nikoobakht and El-Sayed, 2003). CTAB solution $(3.5 \mathrm{~mL}$, $0.14 \mathrm{M})$ was mixed with of $\mathrm{HAuCl}_{4}(0.125 \mathrm{~mL}, 15 \mathrm{mM})$. A freshly prepared, ice-cold $\mathrm{NaBH}_{4}$ solution $(0.50 \mathrm{~mL}, 0.010 \mathrm{M})$ was injected to the mixture, resulting in the formation of a brownish yellow solution. The seed solution was vigorously stirred for $120 \mathrm{~s}$ and then kept at $25^{\circ} \mathrm{C}$.

\section{Synthesis of Au NOPs}

The aqueous solutions of $\mathrm{HAuCl}_{4}(15 \mathrm{mM}, 0.38 \mathrm{~mL}), \mathrm{CTAB}$ $(0.40 \mathrm{M}, 2.375 \mathrm{~mL})$, and 1-MP $(1.0 \mathrm{M}, 0.90 \mathrm{~mL})$ were added in a glass vial, named solution A. Deionized water $(6.12 \mathrm{~mL}), \mathrm{AA}$ $(0.10 \mathrm{M}, 225 \mu \mathrm{L})$, and seeds solution $(1.8 \mu \mathrm{L})$ were added in another glass vial, named solution B. Solution B was quickly added to solution $\mathrm{A}$, and the reaction mixture was incubated at $25^{\circ} \mathrm{C}$ for $5 \mathrm{~min}$.

\section{PEGylation of Au NOPs}

The as-synthesized NOPs $(1.0 \mathrm{~mL})$ were purified by centrifugation at $9,000 \mathrm{rpm}$ for $15 \mathrm{~min}$. The precipitation was redispersed by $1.0 \mathrm{~mL}$ aqueous solution of mPEG-SH $(0.10$ $\mathrm{mg} / \mathrm{mL}$ ). The mixture was ultra-sonicated for $5 \mathrm{~min}$ and stirred over night at room temperature.

\section{Materials Characterization}

The size, morphology, and structure of NOPs were studied by using JEOL JEM-2100F and Hitachi H800 transmission electron microscope (TEM) with an accelerating voltage of 200 and $175 \mathrm{kV}$, respectively. The UV-Vis-NIR extinction spectra were recorded using a Lambda 950 (PerkinElmer) Spectrometer. The purification of NOPs was performed by using Eppendorf Centrifuge $5430 \mathrm{R}$ at $25^{\circ} \mathrm{C}$. X-Ray diffraction (XRD) measurements of NOPs solutions drop-coated onto glass substrates were done on Empyrean (PANalytical B. V.) operating at a voltage of $40 \mathrm{kV}$.

\section{Simulation of the Capping Effect of 1-MP}

Density Functional Theory calculations were performed by means of the Vienna Ab Initio Simulation Package (VASP) (Hafner, 2008) using the Perdew-Burke-Ernzerhof (PBE) generalized-gradient approximation (Perdew et al., 1996). The interactions between valence electrons and ion cores were treated by Blöchl's all-electron-like projector augmented wave (PAW) 
method (Blöchl, 1994). The plane-wave cutoff for the wave functions was $400 \mathrm{eV}$ throughout. For all calculations, we used a $(4 \times 4)$ supercell consisting of a four-layer slab with vacuum thickness of about $20 \AA$ and molecules adsorbed on one side of the slab. The adsorbed molecule and the top three layers of $\mathrm{Au}$ were allowed to relax. The Brillouin zone integration was performed using a cell size dependent Monkhorst-Pack $k$ point sampling (Monkhorst and Pack, 1976) and the $k$-point mesh was $3 \times 3 \times 1$ for surface reconstruction and $7 \times 7 \times$ 1 for single-molecule adsorption. Ionic relaxation for all stable structures was carried out until all forces were smaller than 0.02 $\mathrm{eV} / \mathrm{A}$. The adsorption energy for molecules on Au was calculated as follows:

$$
\Delta E_{a d s}=E_{\text {molecule } / A u}-E_{\text {molecule }}-E_{\text {Au }}
$$

where $E_{\text {molecule } / A u}$ is the total energy of the Au surface together with the adsorbed molecule, $E_{\text {molecule }}$ is the total energy of the free molecule, and $E_{\mathrm{Au}}$ is the total energy of the bare Au surface. With this method, the negative values of adsorption energy suggest that the adsorption configuration is more stable than the corresponding bare Au surface and the free molecule.

\section{Laser-Induced Heat Conversion of Au NOPs}

A diode laser at $660 \mathrm{~nm}$ (LEO photonics Co. Ltd.) was employed in the experiments. PEG-modified NOPs $(1.0 \mathrm{~mL})$ in a quartz cuvette (1-cm path length) was irradiated by the laser with tunable power densities $\left(0.50-2.0 \mathrm{~W} / \mathrm{cm}^{2}\right)$. PEG-modified NOPs with different optical densities (ODs) $(0.10-1.0)$ were irradiated by the laser at $1.0 \mathrm{~W} / \mathrm{cm}^{2}$. Deionized water was used as a negative control. The laser spot was adjusted to cover the entire surface of the sample. Real-time thermal imaging and the maximum temperature was recorded by a FLIR A310 infrared camera.

\section{In vitro Photoacoustic Imaging of Au NOPs}

PEG-modified NOPs solutions with different optical densities were introduced into agar-gel cylinders and put in deionized water at a consistent depth. Photoacoustic images were obtained at $680 \mathrm{~nm}$ wavelength by MSOT inVision 128 small animal scanner.

\section{RESULTS AND DISCUSSION}

The NOPs were prepared by the regrowth from well-defined single-crystalline Au seeds (see section Materials and Methods for details). In a typical synthesis, a seed solution of Au seeds and ascorbic acid (AA) were quickly added into a fresh-prepared growth solution containing $\mathrm{HAuCl}_{4}$ and 1-methylpyrrolidine (1$\mathrm{MP}$ ) in the presence of hexadecyltrimethylammonium bromide $(\mathrm{CTAB})$ at room temperature and final $\mathrm{pH}$ of 11.3. Both AA and 1-MP (Newman and Blanchard, 2006) were reducing agents for $\mathrm{HAuCl}_{4}$, and the basic $\mathrm{pH}$ increased the reduction rates (vide infra). CTAB was used as a surface capping agent to provide the colloidal stability for the synthesized nanoparticles. After the mixing, the color of the solution immediately turned from colorless to peacock blue in about $5 \mathrm{~min}$, indicating the formation of NOPs. Transmission electron microscopy (TEM) study (Figure 1A) of NOPs reveals the NOPs with a high yield (81\%) of possessed eight arms with a uniform arm length and width of $19.5 \pm 1.4$ and $9.4 \pm 1.0 \mathrm{~nm}$, respectively. The NOPs also showed an extraordinary LSPR peak at $680 \mathrm{~nm}$ with a FWHM (Full width at half maximum) as narrow as $76 \mathrm{~nm}(0.20 \mathrm{eV})$ (Mets et al., 2012), which demonstrates high monodispersity of the NOPs (Figure 1B).

Figure 2A shows a high-resolution TEM image of an individual NOP, where periodic lattice fringes can be clearly resolved. The distance between adjacent lattice fringes was about $0.240 \mathrm{~nm}$, corresponding to the d-spacing of $\mathrm{Au}\{111\}$ planes. The X-ray diffraction (XRD) pattern recorded on a glass substrate was displayed in Figure 2B. The diffraction peaks were assigned to (111), (200), (220), (311), (222) planes of face-centered cubic $\mathrm{Au}$, respectively (JCPDS No. 04-0784). It is worth noting that although the ratio between the intensities of the (200) to (111) diffraction peaks was similar to the conventional values $(0.50$ vs. 0.52$)$, the ratios of $(220) /(111)$ and $(220) /(200)$ were significantly lower than the conventional values $(0.21$ vs. 0.32$)$ and $(0.42$ vs. 0.62$)$, respectively. This result indicates that the NOPs were abundant in (111) and (200) facets. Close inspection and analysis of the image (Figure $2 \mathbf{A}$ ) reveals that the arms grew from the eight corners of $\mathrm{Au}$ seeds along $<111>$ directions to form octopod structure. To further understand the spatial arrangement of the arms, three dimensional (3D)-TEM images taken from various tilting angles were consistent with our model of NOPs at different views (Figures 2C,D).

The formation of anisotropic structure requires breaking the face-centered cubic symmetry of Au crystals. Therefore, the growth mechanism is important for understanding how the arms arise from Au seeds. In general, thermodynamically controlled nanocrystals with the global minimum in Gibbs free energy can be synthesized by capping agents which effectively reduce the surface free energy of the nanocrystals. To help elucidate this process, we first considered the effect of CTAB and 1-MP as capping agents. Density Functional Theory (DFT) calculations of the adsorption energy of 1-MP on different Au crystal facets indicate that 1-MP taking a flat conformation with the $\mathrm{N}$ atom preferentially adsorbed on the top-position of $\mathrm{Au}$ atoms (Figure S1). The sequence of 1-MP adsorption energies $\left(\Delta E_{\mathrm{ads}}\right)$ on different crystallographic facets is in agreement with that of CTAB (Liu et al., 2011), i.e., $\left|\Delta E_{\text {ads }}(111)\right|<\left|\Delta E_{\text {ads }}(100)\right|<$ $\left|\Delta E_{\text {ads }}(110)\right|$. This result suggests that the NOPs covered by both 1-MP and CTAB would thermodynamically enclosed by higher proportion of $\{110\}$ facets on the surface, which is conflicting with our analysis of XRD result (Figure 2B).

Our success in the synthesis of NOPs essentially relied on the kinetic control of growth process (Zhang, H. et al., 2010; Xia et al., 2013, 2015; Wang et al., 2015a). As proposed previously, for seed-mediated growth method, the ratio between the deposition rate $\left(V_{\text {dep }}\right)$ and the diffusion rate $\left(V_{\text {diff }}\right)$ determines the growth pathway from a single-crystal seed and thereby the morphology of the produced nanocrystals. $V_{\text {dep }}$ is the rate for the atoms added to the active nanocrystal facets with a higher surface free energy, and $V_{\text {diff }}$ is the rate for the adatoms migrating to the nanocrystal facets with a lower surface free energy. For a 


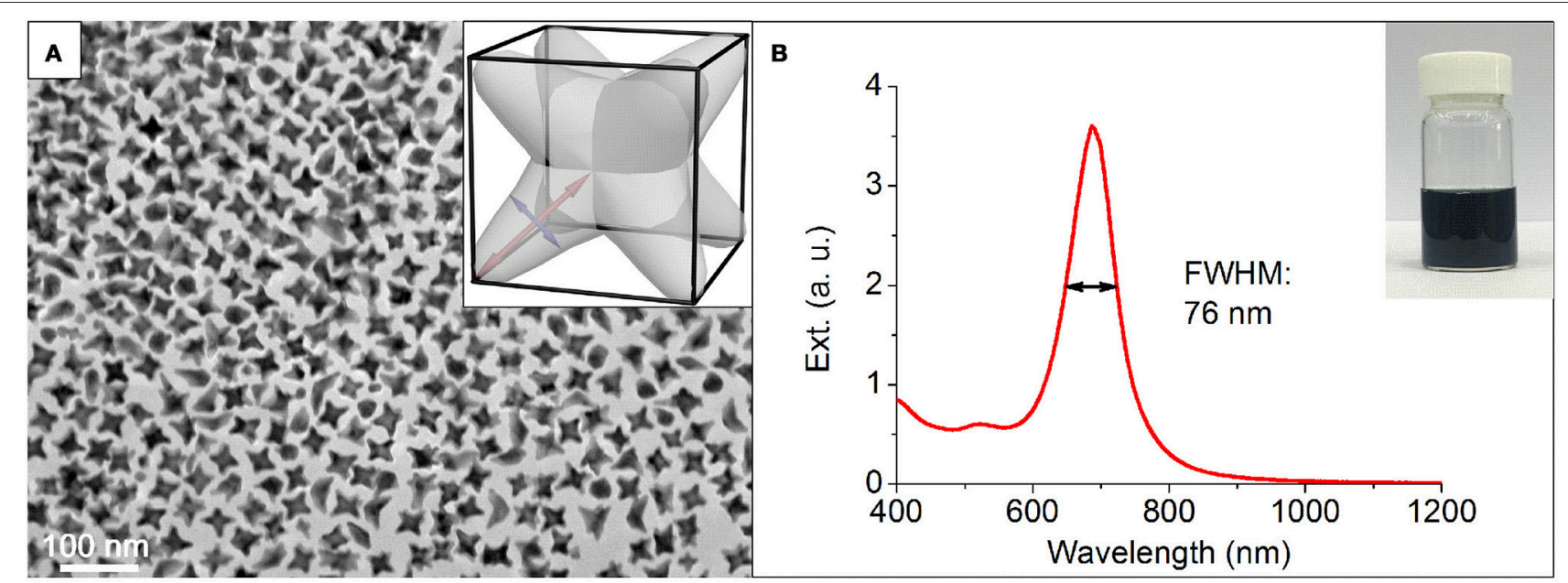

FIGURE 1 | (A) Representative TEM image and (B) extinction spectra of NOPs. Inset in (A): scheme of an individual NOP. Red and blue lines represent the arm length and width, respectively. Inset in (B): optical photograph of the NOP solution.
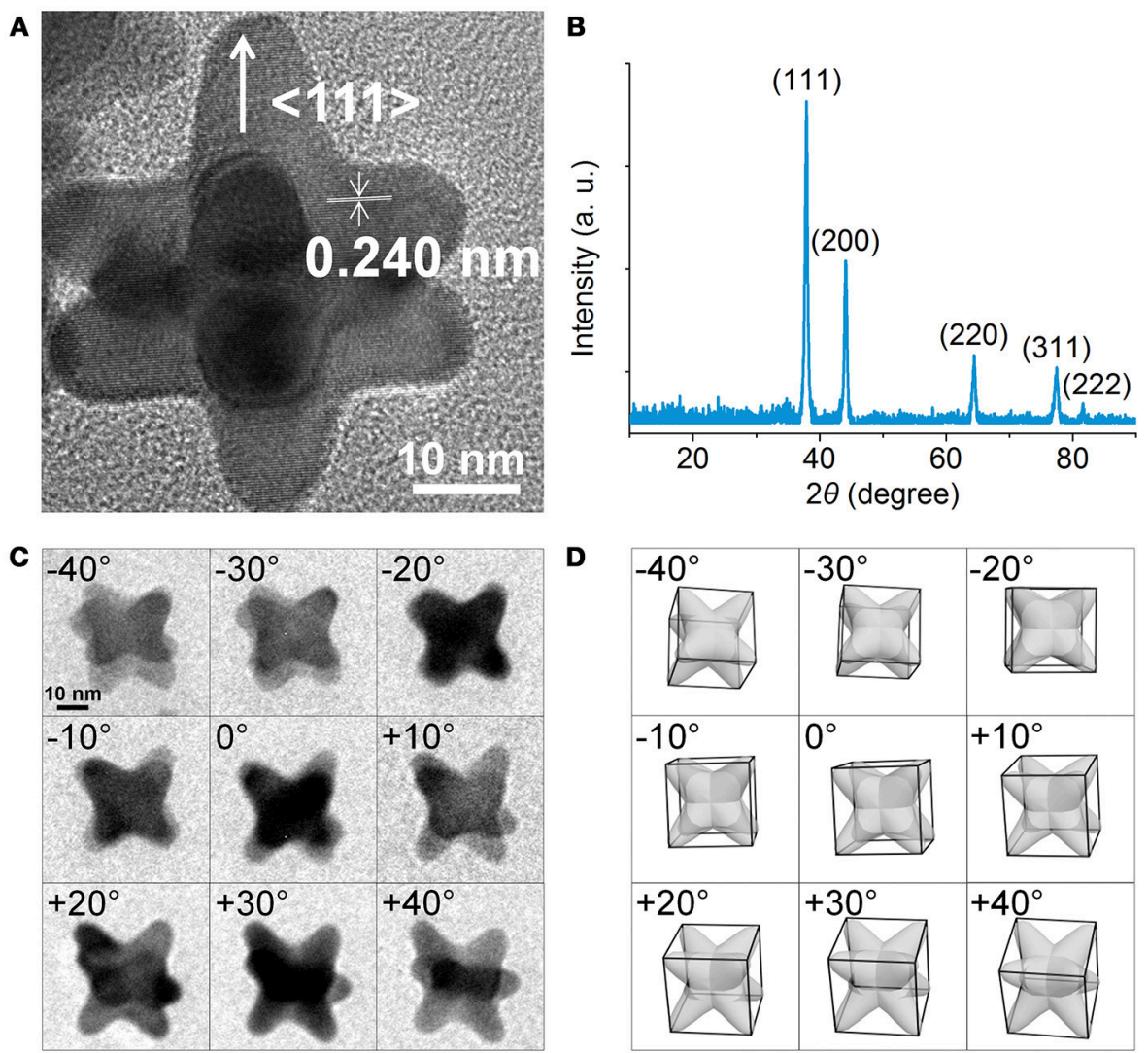

FIGURE 2 | (A) HRTEM image of an individual NOP. (B) Powder X-ray Diffraction of the NOPs. (C) 3 D-TEM images of NOPs tilted from ca. $-40^{\circ}$ to $+40^{\circ}$. (D) Corresponding $3 \mathrm{D}$ model images with same views from ca. $-40^{\circ}$ to $+40^{\circ}$.

near rhombicuboctahedron-shaped single-crystal seed, due to the preferential binding of CTAB capping agent on the $\{100\}$ and $\{110\}$ facets, $\{111\}$ corner facets are the most active sites as a result of less coverage by the capping agent (Park et al., 2013). For a slow reduction rate, $V_{\text {dep }} \ll V_{\text {diff, }}$, most of the adatoms at the corners will migrate to edges and side facets, and the growth will prevail along the $<100>$ and $<110>$ directions, leading to the formation of Wulff shape (Xia et al., 2015), as favored by thermodynamics 
(Figure 3, Path A). On the contrary, for a fast reduction rate, $V_{\text {dep }}$ $\gg V_{\text {diff, }}$, surface diffusion can be ignored and the growth will be switched to the $<111>$ directions, promoting the formation of a kinetically favored octopods as the product (Figure 3, Path B).

Both of $V_{\text {dep }}$ and $V_{\text {diff }}$ are kinetic parameters and can be manipulated by varying the ratio between $\mathrm{Au}$ seeds and $\mathrm{HAuCl}_{4}$, and the rate of reduction of $\mathrm{HAuCl}_{4}$. We studied the reductive ability of AA and 1-MP in the absence of Au seeds by monitoring the peak at $395 \mathrm{~nm}$ which is attributed from the $[\mathrm{CTA}]^{+}$ $\left[\mathrm{AuBr}_{4}\right]^{-}$complex (Kundu, 2013). It is well-known that AA can reduce $\mathrm{HAuCl}_{4}$ to $\mathrm{Au}^{+}$under neutral or acidic condition, but to $\mathrm{Au}^{0}$ under strong alkaline condition (Goia and Matijević, 1999). In the present study when only AA was added into the solution A containing $[\mathrm{CTA}]^{+}\left[\mathrm{AuBr}_{4}\right]^{-}$complex at the $\mathrm{pH}$ of $11.4, \mathrm{Au}$ nanoparticles with a LSPR peak around $530 \mathrm{~nm}$ were formed in $1 \mathrm{~min}$. At the meantime, the peak at $395 \mathrm{~nm}$ was remained (Figure S2A), indicating that under strong alkaline condition, AA could not quickly reduce $\mathrm{Au}^{3+}$ to $\mathrm{Au}^{+}$as it does under neutral or acid condition. The $[\mathrm{CTA}]^{+}\left[\mathrm{AuBr}_{4}\right]^{-}$complex was completely consumed after $3 \mathrm{~h}$, accompanying by the formation of more $\mathrm{Au}$ nanoparticles with quasi-spheres (Figure S3A). This result suggests under strong alkaline condition, although AA can reduce $\mathrm{Au}^{3+}$ to form $\mathrm{Au}$ nanoparticles, the reduction rate of $\mathrm{Au}^{3+}$ to $\mathrm{Au}^{0}$ is slow.

On the other side, when only 1-MP was used as reducing agent under the same condition, the peak of $[\mathrm{CTA}]^{+}\left[\mathrm{AuBr}_{4}\right]^{-}$ complex was completely vanished in $1 \mathrm{~min}$, while no $\mathrm{Au}$ nanoparticles were formed as indicated from the UV-Vis-NIR spectra (Figure S2B). The formation of Au nanoparticles was much slower compared to that of AA. This result reveals that

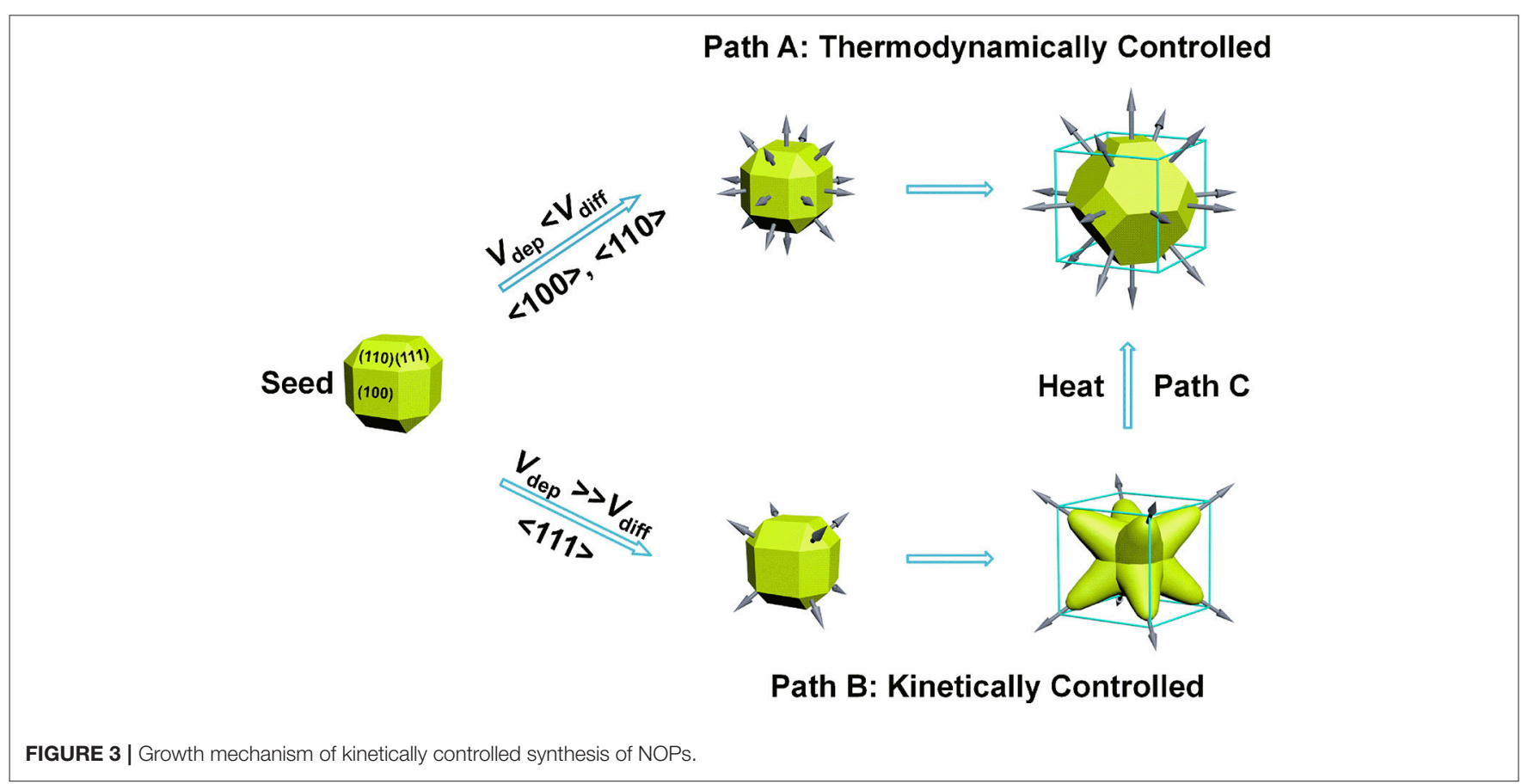

FIGURE 3 | Growth mechanism of kinetically controlled synthesis of NOPs.
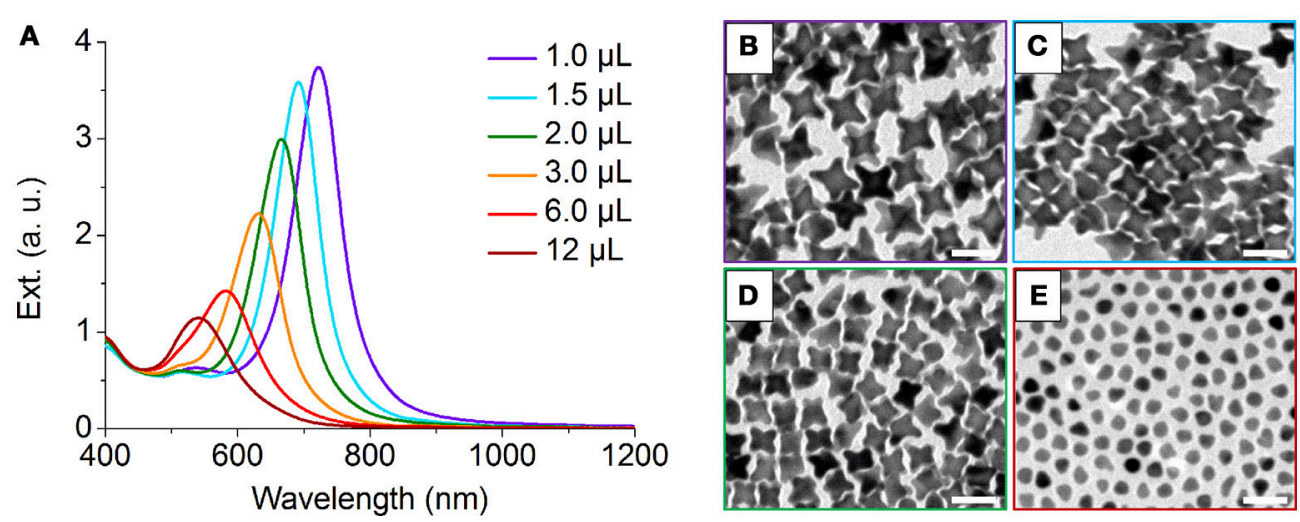

FIGURE 4 | (A) UV-Vis-NIR spectra of NOPs by increasing the volume of Au seeds solution from 1.0 to $12 \mu \mathrm{L}$ added to the growth solution (total volume of $10 \mathrm{~mL}$ ). (B-E) TEM images of NOPs with 1.0, 1.5, 2.0, and $12 \mu \mathrm{L}$ Au seeds solution added to the growth solution, respectively. Scale bars: $50 \mathrm{~nm}$. 
1-MP is a reducing agent which can reduce $\mathrm{Au}^{3+}$ to $\mathrm{Au}^{+}$with a much higher rate compared to AA under basic conditions, but its ability to further reduce $\mathrm{Au}^{+}$to $\mathrm{Au}^{0}$ is weaker than that of AA under strong alkaline condition. From the above results we can conclude that if only AA or 1-MP is used as the solo reducing agent, the reduction rate of formation of $\mathrm{Au}^{0}$ is not high enough to achieve $V_{\text {dep }} \gg V_{\text {diff, }}$ and only spherical or irregular shaped nanoparticles were obtained, respectively (Figure S3). In contrast, when both AA and 1-MP were used, 1-MP can quickly reduce $\mathrm{Au}^{3+}$ to $\mathrm{Au}^{+}$, which can be further reduced to $\mathrm{Au}^{0}$ by $\mathrm{AA}$ to reach a high $V_{\text {dep. Therefore, we }}$ conclude that under the strong alkaline condition, AA and 1MP have a synergistic reducing effect for achieving the condition

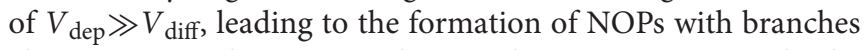
along $<111>$ directions. This result is consistent with the theoretical prediction for the scenario of $V_{\text {dep }} \gg V_{\text {diff }}$ mentioned previously (Xia et al., 2015).

We also performed control experiments to investigate the effect of seed/precursor ratio on the morphology of NOPs. By increasing the volume of $\mathrm{Au}$ seeds solution from 1.0 to $3.0 \mu \mathrm{L}$ with other conditions unchanged, both of the average aspect ratios and lengths of the arms of NOPs can be continuously adjusted from 2.00 to 1.25 and 22.8 to $15.8 \mathrm{~nm}$, respectively (Figure 4, Table S1), that is, the more Au seeds the smaller arms. Further increasing the amount of Au seeds to $12 \mu \mathrm{L}$ led to only thermodynamically stable product, i.e., quasi-spheres
(Figure 4E). This result reveals that when the number of $\mathrm{Au}$ atoms available for every seed is smaller, the ratio of $V_{\text {dep }} / V_{\text {diff }}$ was reduced, leading to thermodynamic products. In addition, this result also demonstrates that one can control the aspect ratio and arm length, as well as LSPR position, by tuning the seed/HAuCl${ }_{4}$ ratio. It is worth mentioning that the arm length and aspect ratio of NOPs depend on their LSPR position, and the LSPR wavelength was linearly correlated with the aspect ratio of NOPs $\left(R^{2}=0.969\right)$ (Figure S4).

Additionally, the reaction temperature also plays a paramount role in determining whether thermodynamic or kinetic control is dominant. High temperatures normally lead to generate thermodynamically controlled nanocrystals. After incubated at higher temperature $\left(60^{\circ} \mathrm{C}\right)$ for $4 \mathrm{~h}$, the morphology of assynthesized NOPs was quickly transformed to Wulff shape with lower surface free energy (Figure S5), which is corresponding to the path $\mathrm{C}$ in Figure 3. This result confirms the NOPs are kinetically controlled product, of which the shape changes to thermodynamically stable at high temperatures.

It's worth pointing out that the NOPs are thermodynamically unstable due to a relatively high surface energy at the sharp tips, resulting in changes of their morphology to less anisotropic structures and the blue-shifting of their LSPR bands (Figure S6; Wang et al., 2015b). Therefore, the practical applications of the NOPs are severely limited. In search of the appropriate methods for stabilizing the NOPs, we have tried halogen ions, surfactants
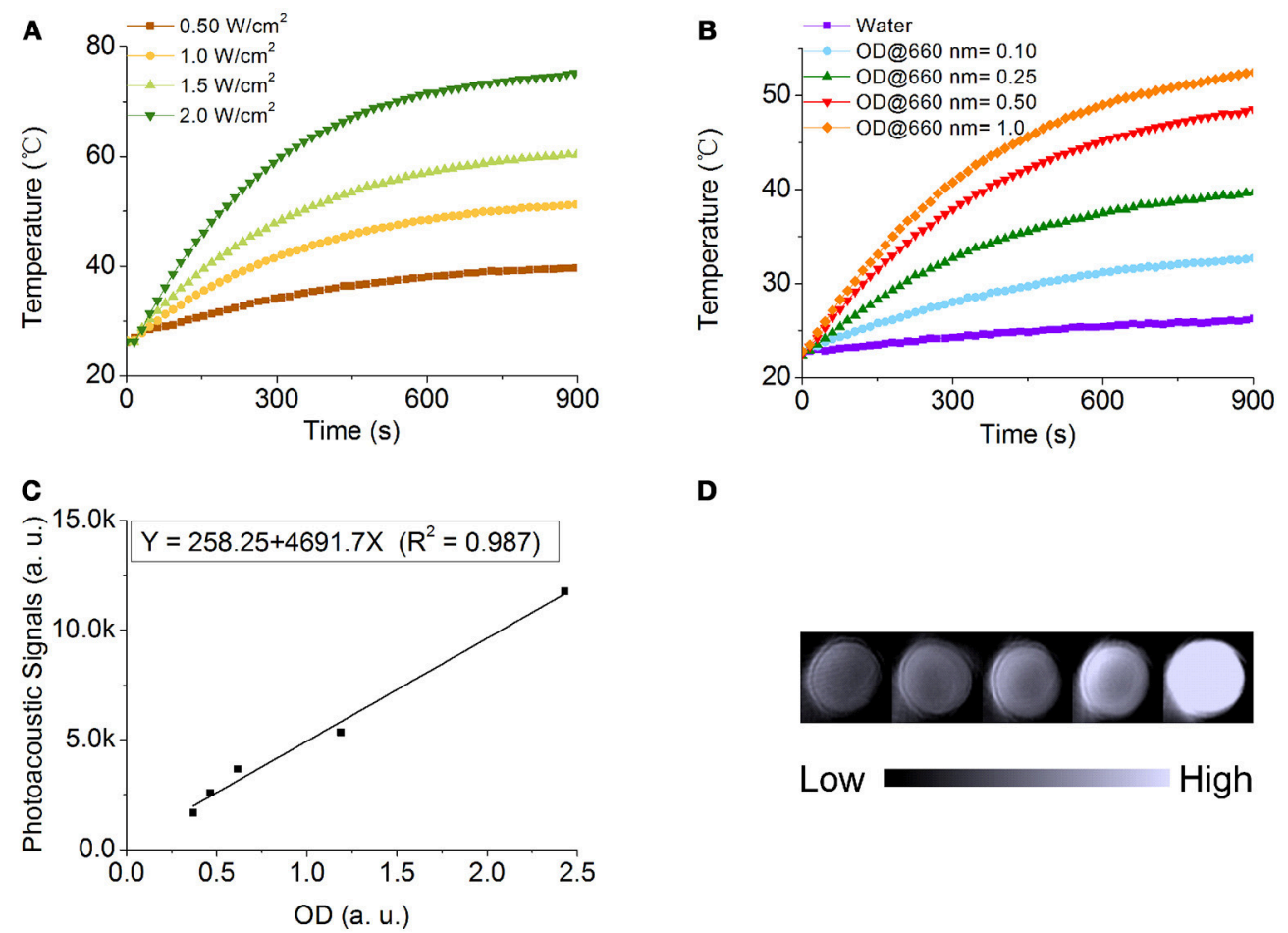

D

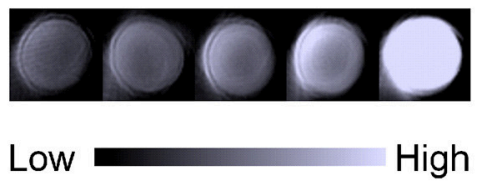

FIGURE 5 | Photothermal conversion and photoacoustic properties of NOPs. (A) Temperature variation of NOPs with the optical density at $660 \mathrm{~nm}\left(\mathrm{OD}_{660 \mathrm{~nm}}\right)$ of 1.0 irradiated at different laser power densities: 0.50 (brown), 1.0 (yellow), 1.5 (light green), and $2.0 \mathrm{~W} / \mathrm{cm}^{2}$ (dark green). (B) Temperature variation of NOPs with different OD $660 n m$ values: 0.10 (blue), 0.25 (green), 0.50 (red), and 1.0 (orange) irradiated at a laser power density of 1.0 W/cm². (C) Photoacoustic signals of NOPs as a function of $\mathrm{OD}_{680 \mathrm{~nm}}$. (D) Photoacoustic images of NOPs. 
(i.e., CTAB), and six thiol compounds, including cysteine, 3-mercaptopropionic acid, glutathione, 11-mercaptopropionic acid, polyetherimide, mPEG-SH. Among them, we found that mPEG-SH modified NOPs showed negligible shifting of their LSPR band in 5 days at room temperature (Figure S7) and good thermal-stability at high temperature (at $75^{\circ} \mathrm{C}$ for $30 \mathrm{~min}$ ) (Figure S8).

Owing to the strong LSPR band at $680 \mathrm{~nm}$ of NOPs, it is worthy to evaluate their dual potential as photothermal conversion and photoacoustic contrast agents. To evaluate their photothermal conversion performance, aqueous solutions of PEG-modified NOPs with the same optical density at $660 \mathrm{~nm}\left(\mathrm{OD}_{660 \mathrm{~nm}}\right)$ of 1.0 were exposed to the $660 \mathrm{~nm}$ laser at different power densities $\left(0.50-2.0 \mathrm{~W} / \mathrm{cm}^{2}\right)$ as shown in Figure 5A. Additionally, PEG-modified NOPs with different optical densities (ODs) (0.10-1.0) were irradiated by the laser at $1.0 \mathrm{~W} / \mathrm{cm}^{2}$ (Figure 5B). After the laser irradiation, PEGmodified NOPs showed negligible shifting of their LSPR band and kept their morphology unchanged as well (Figure S9). The temperature variation was strongly depended on the laser power and concentration of NOPs (Figures 5A,B). Table S2 lists the photothermal conversion values of NOPs calculated by using a modified model similar to the report by Roper et al. (Roper et al., 2007; Shao et al., 2016). NOPs showed excellent photothermal conversion efficiencies with a maximum value up to $83.0 \%$. The ultrahigh conversion value is attributed to the narrow LSPR band of NOPs. In addition, the solution of NOPs also presented a strong photoacoustic signal at $680 \mathrm{~nm}$ wavelength. The photoacoustic intensity was linearly proportional to the concentration of NOPs with $R^{2}=0.987$, suggesting the NOPs as a promise photoacoustic contrast agent.

\section{CONCLUSION}

Monodisperse NOPs were synthesized through a kinetically controlled growth with a high yield and ultra-narrow LSPR band width. The synergistic reducing effects of AA and 1-MP

\section{REFERENCES}

Averitt, R. D., Sarkar, D., and Halas, N. J. (1997). Plasmon resonance shifts of $\mathrm{Au}$-coated $\mathrm{Au}_{2} \mathrm{~S}$ nanoshells: insight into multicomponent nanoparticle growth. Phys. Rev. Lett. 78, 4217-4220. doi: 10.1103/PhysRevLett.78.4217

Blöchl, P. E. (1994). Projector augmented-wave method. Phys. Rev. B 50, 17953-17979. doi: 10.1103/PhysRevB.50.17953

Boles, M. A., Ling, D., Hyeon, T., and Talapin, D. V. (2016). The surface science of nanocrystals. Nat. Mater. 15, 141-153. doi: 10.1038/ nmat 4526

Chen, S., Wang, Z. L., Ballato, J., Foulger, S. H., and Carroll, D. L. (2003). Monopod, bipod, tripod, and tetrapod gold nanocrystals. J. Am. Chem. Soc. 125, 16186-16187. doi: 10.1021/ja038927x

Cheng, K., Kothapalli, S. R., Liu, H., Koh, A. L., Jokerst, J. V., Jiang, H., et al. (2014). Construction and validation of nano gold tripods for molecular imaging of living subjects. J. Am. Chem. Soc. 136, 3560-3571. doi: 10.1021/ja 412001e

Dam, D. H. M., Lee, J. H., Sisco, P. N., Co, D. T., Zhang, M., Wasielewski, M. R., et al. (2012). Direct observation of nanoparticle-cancer were important for the kinetically controlled scenario of $V_{\text {dep }}$ $\gg V_{\text {diff }}$, resulting the formation of the highly branched NOPs along eight $<111>$ directions. The uniform of the number, size, and orientation of their branches makes NOPs their remarkable LSPR properties with ultra-narrow LSPR band. The NOPs offer outstanding properties in bio-applications such as photothermal therapy with high photothermal conversion efficiencies up to $83.0 \%$ and a promise photoacoustic contrast agent in photoacoustic imaging. Owing to their excellent LSPR properties, the NOPs are highly promising in diverse applications, such as sensing, nanodevices, catalysis, and bio-applications.

\section{AUTHOR CONTRIBUTIONS}

Y-XC performed the experiments and analyzed the data; H-MG and Z-YL performed the Density Functional Theory calculations; $\mathrm{N}-\mathrm{NZ}$ and $\mathrm{X}$-FT provided assistance in the experiments; $\mathrm{KL}$, BY, JZ, and TS designed the experiments; KL and Y-XC wrote the manuscript. All authors read and approved the manuscript.

\section{ACKNOWLEDGMENTS}

KL thanks the National Natural Science Foundation of China (21474040, 21674042) and China's Thousand Talent Plan for financial support. KL and Z-YL gratefully acknowledge financial support from the National Natural Science Foundation of China (21534004). JZ, KL, and BY thank for the support of JLU Science and Technology Innovative Research Team 2017TD-06. KL and TS thank for Interdisciplinary Innovation Project of the First Hospital of Jilin University (JDYYJCHX001).

\section{SUPPLEMENTARY MATERIAL}

The Supplementary Material for this article can be found online at: https://www.frontiersin.org/articles/10.3389/fchem. 2018.00335/full\#supplementary-material

cell nucleus interactions. ACS Nano 6, 3318-3326. doi: 10.1021/ nn300296p

Goia, D. V., and Matijević, E. (1999). Tailoring the particle size of monodispersed colloidal gold. Colloids Surf. A 146, 139-152. doi: 10.1016/S0927-7757(98)00790-0

Guerrero-Martínez, A., Barbosa, S., Pastoriza-Santos, I., and Liz-Marzán, L. M. (2011). Nanostars shine bright for you: colloidal synthesis, properties and applications of branched metallic nanoparticles. Colloid Interface Sci. 16, 118-127. doi: 10.1016/j.cocis.2010.12.007

Hafner, J. (2008). Ab-initio simulations of materials using VASP: densityfunctional theory and beyond. J. Comput. Chem. 29, 2044-2078. doi: $10.1002 /$ jcc. 21057

Hao, E., Bailey, R. C., Schatz, G. C., Hupp, J. T., and Li, S. (2004). Synthesis and optical properties of "branched" gold nanocrystals. Nano Lett. 4, 327-330. doi: $10.1021 / \mathrm{nl} 0351542$

Hoggard, A., Wang, L. Y., Ma, L., Fang, Y., You, G., Olson, J., et al. (2013). Using the plasmon linewidth to calculate the time and efficiency of electron transfer between gold nanorods and graphene. ACS Nano 7, 11209-11217. doi: $10.1021 / \mathrm{nn} 404985 \mathrm{~h}$ 
Indrasekara, A. S. D. S., Meyers, S., Shubeita, S., Feldman, L. C., Gustafsson, T., and Fabris, L. (2014). Gold nanostar substrates for SERS-based chemical sensing in the femtomolar regime. Nanoscale 6, 8891-8899. doi: 10.1039/C4NR02513J

Jana, N. R., Gearheart, L., and Murphy, C. J. (2001). Wet chemical synthesis of high aspect ratio cylindrical gold nanorods. J. Phys. Chem. B 105, 4065-4067. doi: $10.1021 /$ jp0107964

Kelly, K. L., Coronado, E., Zhao, L. L., and Schatz, G. C. (2003). The optical properties of metal nanoparticles: the influence of size, shape, and dielectric environment. J. Phys. Chem. B 107, 668-677. doi: 10.1021/jp026731y

Khoury, C. G., and Vo-Dinh, T. (2008). Gold nanostars for surface-enhanced Raman scattering: synthesis, characterization and optimization. J. Phys. Chem. C 112, 18849-18859. doi: 10.1021/jp8054747

Kim, F., Connor, S., Song, H., Kuykendall, T., and Yang, P. (2004). Platonic gold nanocrystals. Angew. Chem. 116, 3759-3763. doi: 10.1002/ange.200454216

Kumar, P. S., Pastoriza-Santos, I., Rodríguez-González, B., García de Abajo, F. J., and Liz-Marzán L. M. (2007). High-yield synthesis and optical response of gold nanostars. Nanotechnology 19, 015606-015611. doi: 10.1088/0957-4484/19/01/015606

Kundu, S. (2013). A new route for the formation of Au nanowires and application of shape-selective Au nanoparticles in SERS studies. J. Mater. Chem. C 1, 831-842. doi: 10.1039/C2TC00315E

Li, N., Zhao, P., and Astruc, D. (2014). Anisotropic gold nanoparticles: synthesis, properties, applications, and toxicity. Angew. Chem. Int. Ed. 53, 1756-1789. doi: $10.1002 /$ anie.201300441

Lim, B., and Xia, Y. (2011). Metal nanocrystals with highly branched morphologies. Angew. Chem. Int. Ed. 50, 76-85. doi: 10.1002/anie.201002024

Link, S., and El-Sayed, M. A. (2000). Shape and size dependence of radiative, non-radiative and photothermal properties of gold nanocrystals. Int. Rev. Phys. Chem. 19, 409-453. doi: 10.1080/01442350050034180

Liu, K., Zhao, N., and Kumacheva, E. (2011). Self-assembly of inorganic nanorods. Chem. Soc. Rev. 40, 656-671. doi: 10.1039/c0cs00133c

Liu, M., and Guyot-Sionnest, P. (2005). Mechanism of silver (I)-assisted growth of gold nanorods and bipyramids. J. Phys. Chem. B 109, 22192-22200. doi: $10.1021 /$ jp054808n

Malikova, N., Pastoriza-Santos, I., Schierhorn, M., Kotov, N. A., and Liz-Marzán, L. M. (2002). Layer-by-layer assembled mixed spherical and planar gold nanoparticles: control of interparticle interactions. Langmuir 18, 3694-3697. doi: $10.1021 / \mathrm{la} 025563 \mathrm{y}$

Mayer, K. M., and Hafner, J. H. (2011). Localized surface plasmon resonance sensors. Chem. Rev. 111, 3828-3857. doi: 10.1021/cr100313v

Mets, O. M., de Jong, P. A., van Ginneken, B., Gietema, H. A., and Lammers, J. W. J. (2012). Quantitative computed tomography in COPD: possibilities and limitations. Lung 190, 133-145. doi: 10.1007/s00408-011-9353-9

Monkhorst, H. J., and Pack, J. D. (1976). Special points for Brillouin-zone integrations. Phys. Rev. B 13, 5188-5192. doi: 10.1103/PhysRevB.13.5188

Nehl, C. L., Liao, H., and Hafner, J. H. (2006). Optical properties of star-shaped gold nanoparticles. Nano Lett. 6, 683-688. doi: 10.1021/nl052409y

Newman, J. D. S., and Blanchard, G. J. (2006). Formation of gold nanoparticles using amine reducing agents. Langmuir 22, 5882-5887. doi: 10.1021/la060045z

Nikoobakht, B., and El-Sayed, M. A. (2003). Preparation and growth mechanism of gold nanorods (NRs) using seed-mediated growth method. Chem. Mater. 15, 1957-1962. doi: $10.1021 / \mathrm{cm} 0207321$

Niu, W., Chua, Y. A. A., Zhang, W., Huang, H., and Lu, X. (2015). Highly symmetric gold nanostars: crystallographic control and surfaceenhanced Raman scattering property. J. Am. Chem. Soc. 137, 10460-10463. doi: 10.1021/jacs.5b05321

Park, K., Drummy, L. F., Wadams, R. C., Koerner, H., Nepal, D., Fabris, L., et al. (2013). Growth mechanism of gold nanorods. Chem. Mater. 25, 555-563. doi: $10.1021 / \mathrm{cm} 303659 \mathrm{q}$

Perdew, J. P., Burke, K., and Ernzerhof, M. (1996). Generalized gradient approximation made simple. Phys. Rev. Lett. 77, 3865-3868. doi: 10.1103/PhysRevLett.77.3865

Roper, D. K., Ahn, W., and Hoepfner, M. (2007). Microscale heat transfer transduced by surface plasmon resonant gold nanoparticles. J. Phys. Chem. C 111, 3636-3641. doi: 10.1021/jp064341w

Shankar, S. S., Rai, A., Ankamwar, B., Singh, A., Ahmad, A., and Sastry, M. (2004). Biological synthesis of triangular gold nanoprisms. Nat. Mater. 3, 482-488. doi: $10.1038 /$ nmat1 152
Shao, J., Xie, H., Huang, H., Li, Z., Sun, Z., Xu, Y. et al. (2016). Biodegradable black phosphorus-based nanospheres for in vivo photothermal cancer therapy. Nat. Commun. 7, 12967-12979. doi: 10.1038/ncomms 12967

Skrabalak, S. E., Chen, J., Sun, Y., Lu, X., Au, L., Cobley, C. M., et al. (2008). Gold nanocages: synthesis, properties, and applications. Acc. Chem. Res. 41, 1587-1595. doi: 10.1021/ar800018v

Smith, A. F., Weiner, R. G., and Skrabalak, S. E. (2016). Symmetry-dependent optical properties of stellated nanocrystals. J. Phys. Chem. C 120, 20563-20571. doi: $10.1021 /$ acs.jpcc. 5 b12280

Sönnichsen, C., Franzl, T., Wilk, T., von Plessen, G., Feldmann, J., Wilson, O., et al. (2002). Drastic reduction of plasmon damping in gold nanorods. Phys. Rev. Lett. 88, 077402. doi: 10.1103/PhysRevLett.88.077402

Sun, Y., and Xia, Y. (2002). Shape-controlled synthesis of gold and silver nanoparticles. Science 298, 2176-2179. doi: 10.1126/science.1077229

Wang, Y., Black, K. C. L., Luehmann, H., Li, W., Zhang, Y., Cai, X., et al. (2013). Comparison study of gold nanohexapods, nanorods, and nanocages for photothermal cancer treatment. ACS Nano 7, 2068-2077. doi: $10.1021 / \mathrm{nn} 304332 \mathrm{~s}$

Wang, Y., He, J., Liu, C., Chong, W. H., and Chen, H. (2015a). Thermodynamics versus kinetics in nanosynthesis. Angew. Chem. Int. Ed. 54, 2022-2051. doi: 10.1002/anie.201402986

Wang, Y., Serrano, A. B., Sentosun, K., Bals, S., and Liz-Marzán, L. M. (2015b). Stabilization and encapsulation of gold nanostars mediated by dithiols. Small 11, 4314-4320. doi: 10.1002/smll.201500703

Wei, Q., Song, H. M., Leonov, A. P., Hale, J. A., Oh, D., Ong, Q. K., et al. (2009). Gyromagnetic imaging: dynamic optical contrast using gold nanostars with magnetic cores. J. Am. Chem. Soc. 131, 9728-9734. doi: 10.1021/ja901562j

Xia, X., Xie, S., Liu, M., Peng, H. C., Lu, N., Wang, J., et al. (2013). On the role of surface diffusion in determining the shape or morphology of noble-metal nanocrystals. Proc. Natl. Acad. Sci. U.S.A. 110, 6669-6673. doi: 10.1073/pnas. 1222109110

Xia, Y., Xia, X., and Peng, H. C. (2015). Shape-controlled synthesis of colloidal metal nanocrystals: thermodynamic versus kinetic products. J. Am. Chem. Soc. 137, 7947-7966. doi: 10.1021/jacs.5b04641

Ye, E., Regulacio, M. D., Zhang, S. Y., Loh, X. J., and Han, M. Y. (2015). Anisotropically branched metal nanostructures. Chem. Soc. Rev. 44, 6001-6017. doi: 10.1039/C5CS00213C

Yuan, H., Fales, A. M., and Vo-Dinh, T. (2012). TAT peptide-functionalized gold nanostars: enhanced intracellular delivery and efficient NIR photothermal therapy using ultralow irradiance. J. Am. Chem. Soc. 134, 11358-11361. doi: $10.1021 /$ ja304180y

Zhang, H., Li, W., Jin, M., Zeng, J., Yu, T., Yang, D., et al. (2010). Controlling the morphology of rhodium nanocrystals by manipulating the growth kinetics with a syringe pump. Nano Lett. 11, 898-903. doi: 10.1021/nl104347j

Zhang, J., Langille, M. R., Personick, M. L., Zhang, K., Li, S., and Mirkin, C. A. (2010). Concave cubic gold nanocrystals with high-index facets. J. Am. Chem. Soc. 132, 14012-14014. doi: 10.1021/ja106394k

Zhu, X., Zhuo, X., Li, Q., Yang, Z., and Wang, J. (2016). Gold nanobipyramidsupported silver nanostructures with narrow plasmon linewidths and improved chemical stability. Adv. Funct. Mater 26, 341-352. doi: $10.1002 / \mathrm{adfm} .201503670$

Zijlstra, P., Paulo, P. M. R., Yu, K., Xu, Q. H., and Orrit, M. (2012). Chemical interface damping in single gold nanorods and its near elimination by tip-specific functionalization. Angew. Chem. Int. Ed. 51, 8352-8355. doi: $10.1002 /$ anie. 201202318

Conflict of Interest Statement: The authors declare that the research was conducted in the absence of any commercial or financial relationships that could be construed as a potential conflict of interest.

Copyright $\odot 2018$ Chang, Gao, Zhang, Tao, Sun, Zhang, Lu, Liu and Yang. This is an open-access article distributed under the terms of the Creative Commons Attribution License (CC BY). The use, distribution or reproduction in other forums is permitted, provided the original author(s) and the copyright owner(s) are credited and that the original publication in this journal is cited, in accordance with accepted academic practice. No use, distribution or reproduction is permitted which does not comply with these terms. 